\title{
Gain-Loss Framing Enhances Mnemonic Discrimination in Preschoolers
}

\author{
Chi T. Ngo (D), Nora S. Newcombe, and Ingrid R. Olson \\ Temple University
}

\begin{abstract}
Episodic memory relies on discriminating among similar elements of episodes. Mnemonic discrimination is relatively poor at age 4 , and then improves markedly. We investigated whether motivation to encode items with fine-grain resolution would change this picture of development, using an engaging computer-administered memory task in which a bird ate items that made her healthier (gain frame), sicker (loss frame), or led to no change (control condition). Using gain-loss framing led to enhanced mnemonic discrimination in 4- and 5-year-olds, but did not affect older children or adults. Despite this differential improvement, age-related differences persisted. An additional finding was that loss-framing led to greater mnemonic discrimination than gain-framing across age groups. Motivation only partially accounts for the improvement in mnemonic discrimination.
\end{abstract}

\section{Mnemonic Discrimination Development}

Memories for everyday events share a great deal of overlap, as our experiences often take place in similar places and involve similar objects and people with similar characteristics. Therefore, episodic memory requires successful mnemonic discrimination of people, places and objects, and the ability to encode and store past events with a high degree of specificity. For example, if you went mushroom picking and ate a mushroom that made you violently ill, it is important to be able to remember and later discriminate the sickness-inducing mushroom from the good mushrooms. In instances in which the outcomes of consuming two different types of mushrooms are undifferentiated, mnemonic discrimination between these similar experiences may be less behaviorally important. Mnemonic discrimination may involve a process called pattern separation, a hippocampal computation that reduces the degree of overlap between similar inputs to circumvent catastrophic interference (Complementary Learning Systems theory: Norman \& O'Reilly, 2003; O'Reilly \& McClelland,

We thank Ying Lin, Elizabeth Eberts, Jelani Mumford, Jessica Palmarini, and Ai Leen Oon, for their help with stimuli development and data collection, and Maria Brucato for the voice recording of the memory task. We acknowledge support from the National Institutes of Health (F31HD090872 to Chi T. Ngo), although the content is solely the responsibility of the authors and does not necessarily represent the official views of the National Institute of Mental Health. The authors declare no competing or conflicting financial interests.

Correspondence concerning this article should be addressed to Chi T. Ngo, Department of Psychology, Temple University, 1701 N. 13th Street, Philadelphia, PA 19122. Electronic mail may be sent to chi.ngo@temple.edu.
1994). A subfield of the hippocampus called the dentate gyrus (DG) is thought to perform this function by assigning distinct representations to highly overlapping inputs, reducing catastrophic interference among similar experiences. One behavioral expression of pattern separation is the ability to remember similar, but not identical items, memories as distinct from one another, that is, lure discrimination (reviewed in Yassa \& Stark, 2011).

Recent findings indicate that mnemonic discrimination is quite poor during preschool ages, and improves markedly throughout early and middle childhood (Canada, Ngo, Newcombe, Geng, \& Riggins, 2018; Ngo, Newcombe, \& Olson, 2018; Rollins \& Cloude, 2018), coinciding with the developmental window in which episodic memory shows the most robust gains (reviewed in Ghetti \& Bunge, 2012; Olson \& Newcombe, 2014). One paradigm designed to test mnemonic discrimination is the Mnemonic Similarity Task (MST), in which studied objects must be discriminated from perceptually similar objects at test (reviewed in Yassa \& Stark, 2011). At encoding, participants first view a series of object images. Subsequently, another series of objects are shown, including some that are identical to previously studied items (old), some that are similar exemplars of the studied objects (lure), and some that are dissimilar to the rest of the studied objects (novel). Participants made "old," "similar," and "new" memory judgments for each test object. Mnemonic discrimination is index by participants'

(C) 2019 Society for Research in Child Development All rights reserved. 0009-3920/2019/xxxx-xxxx DOI: $10.1111 /$ cdev.13297 
abilities to correctly identify lures as similar, while correcting for the frequency of misidentifying lures as old items. Four-year-old children consistently showed poorer mnemonic discrimination than 6year-olds and young adults, whereas only subtle differences were detected between 6-year-olds and young adults in a child-friendly version of the MST (Ngo et al., 2018). Similarly, in a 4-alternativeforced-choice task that includes targets and similar lures, 4-year-olds chose similar lures more often than 6-year-olds and young adults (Ngo, Lin, Newcombe, \& Olson, 2019). The development of mnemonic discrimination may play an important role in the lifting of childhood amnesia (Canada et al., 2018; Keresztes et al., 2017; Ngo et al., 2018).

These findings raise the question: why do young children perform poorly on mnemonic discrimination tasks? One reason may be neurobiological-before the age of 6, late-developing subfields of the hippocampus (i.e., DG), limiting optimal pattern separation, thereby resulting in poor mnemonic discrimination. Canada et al. (2018) found that (a) performance on the MST improved from ages 4 to 8 , (b) C2-4/DG gray matter volume waxed and then waned in this age range (i.e., showed a quadratic relation to age), and (c) crucially, in younger children, greater volume was related to better discrimination, whereas in older children, the opposite was true. Discrimination performance on the MST also correlated linearly with age in a sample of children aged 6-14. Furthermore, discrimination was associated with hippocampal maturation characterized by the multivariate patterns of age-related differences in gray matter volumes of hippocampal subfields (Keresztes et al., 2017).

When young children perform poorly on a task, it is always important to ensure that they comprehended the task demands. Previous studies implemented pretest practice sessions to facilitate children's understanding of the task (Canada et al., 2018; Ngo et al., 2018; Rollins \& Cloude, 2018). Even the youngest group of children (age 4) showed effects of the level of similarity between lures and targets, demonstrating an understanding of the task procedure (Ngo et al., 2018). However, children's abilities to spontaneously form memories with high resolution may not reflect what they can do when motivated to encode and retrieve the specific details of the studied objects. In fact, signals from extra-hippocampal structures that carry information about behavioral significance - emotional, motivational, or attentional signals - can influence pattern separation (reviewed in Kassab \& Alexandre, 2018). Several studies have demonstrated that arousal evoked at encoding may enhance subsequent mnemonic discrimination in young adults (Balderston et al., 2017; Segal, Stark, Kattan, Stark, \& Yassa, 2012). However, there have been no studies investigating the role of motivation on mnemonic discrimination in children.

It has been found that an age-appropriate cover story may sufficiently motivate children in other cognitive domains. For examples, one study examined the impact of the use of reward with an ageappropriate cover story (combatting an alien invasion) to motivate children aged 7-10 in a working memory task (Atkinson, Waterman, \& Allen, 2019). Children showed higher accuracy for the valued items, suggesting that they are able to engage in executive control in order to prioritize high-reward items when sufficiently motivated to do so. Interestingly, the prioritizing effects for high-value items were not observed using a task developed for adults without an adequate adaptation (earning stickers for a reward at the end of each session to make a task age appropriate (Berry, Waterman, Baddeley, Hitch, \& Allen, 2018). Note that explicit reward was used in both studies to probe prioritization, thus the main difference is in the implementation of a compelling cover story that engages children in the task. Taken together, these findings support the idea that employing engaging and the age-appropriate tasks is crucial (Atkinson et al., 2019; Light, Buckingham, \& Robbins, 1979).

This study sought to investigate the malleability of age-related differences in mnemonic discrimination during early and middle childhood. We asked whether motivating children to encode items with high specificity using a gain-loss framing paradigm would affect subsequent mnemonic discrimination. In an adaptive memory system, experiences should be prioritized based on their significance. If we think an event may be important for guiding future behaviors, there is a strong incentive to remember it.

\section{Current Study}

Here we asked whether gain-loss framing would motivate children to encode and/or retrieve past experiences with high resolution. One prediction is that poor mnemonic discrimination in preschoolers is due to low motivation. Thus when sufficiently motivated, they should perform comparably to older children. An alternative prediction is that motivation may boost mnemonic discrimination to a certain degree; however, it would not abolish age-related differences between younger and older children. If true, these findings would suggest that the persistent age-related differences in mnemonic 
discrimination are likely due to immaturity of the neural circuits supporting pattern separation.

To increase motivation for encoding fine-grain details, gain-loss framing was employed such that items were either portrayed as positive or negative in an arbitrary fashion. To do this, we created an engaging game-like computerized task in which children learned that certain food items made the main character sick or healthy in the experimental condition. We included a control condition in which the items were learned in the same cover story, but devoid of the sick/healthy element. We reasoned that children would care about the health of the main character, and this would motivate them to pay attention to the discriminating details of the food items. Given that the age range between 4 and 6 years is a window in which gains in episodic memory are most robust (Peterson, Warren, \& Short, 2011), with relatively less drastic changes occurring from age 6 onward (reviewed in Olson \& Newcombe, 2014), we tested younger children (preschoolers-ages 4-5), older children (ages 6-8), and young adults.

\section{Methods}

\section{Participants}

A total of forty-five 4- and 5-year-old children (20 and 25 in the experimental and control conditions, respectively) and fifty-two 6- to 8-year-old children (29 and 23 in the experimental and control conditions, respectively) recruited from the Philadelphia suburban areas participated in the study at the Temple Ambler Infant and Child Laboratory. All children were free of neurological damage and had no history of developmental disorders as reported by a parent. Two additional children (one 5-year-old and one 7year-old) were tested but did not complete the task. All children received a small toy for their participation. The adult sample consisted of 44 undergraduate students (22 in each condition) from Temple University who participated for partial course credit (see Table 1 for age-related descriptive reports of all age groups). All participants gave informed consent and reported to have normal or corrected-to-normal vision. This experiment was approved by the Institutional Review Board committee at Temple University.

\section{Memory Task}

\section{Materials}

An animation sequence of a forest scene was created in Adobe Photoshop CS6. One GIF image of a cartoon bird was obtained to serve as the character of the cover story for the memory task. Ninety-six images of animate objects (32 triplets for each object exemplar) were selected from a pool of 183 object images sampled from a Google image search engine. The encoding and test instructions were given by prerecorded voice audio. Testing materials have been made publically available (https://osf. io/p7jvf/).

\section{Procedure}

Children. All children were randomly assigned to either the experimental or control condition, and to one of the three animation versions. Children were tested individually. The task procedure was presented on a 13 in. laptop screen, and entailed two encoding - test blocks, with 16 encoding and 16 test trials within each block, totaling 32 encoding and 32 test trials across the entire experiment. In the experimental condition, the female voice recording introduced the cover story at the beginning of the encoding phase by saying, "Meet my friend Birdie! She's taking the trip to the forest. In this forest, there will be some foods she would like to eat. Some foods make her healthy, and some foods make her sick." One example trial for both the healthy and sick conditions was introduced. On the "healthy" example trial, an image of a food (e.g., a leaf) appeared on the screen, whereas the health bar was presented below. The instruction said, "When she eats a food that makes her healthy, her health bar goes up, like this," as a subsequent increase in the health bar was demonstrated. During the "sick" example trial, an image of another type of food (e.g., an apple) was presented, again with the health bar being presented below. On this trial, the voice recording stated, "When she eats a food that makes her sick, her health bar goes down, like this," while a consequent decrease in the health bar ensued. The encoding instructions continued with, "Birdie will eat a lot of food, watch out for the things that make her sick or healthy, ok? Are you ready to start?" Throughout the encoding phase, Birdie was seen flying through the forest, encountering a green diamond at fixed intervals (see Figure 1A). The appearance of the green diamond indicated that there was food that she wanted to eat. The screen zoomed into the diamond, initiating a trial of the encoding phase. In each trial, the image of the object appeared on the screen automatically for $2 \mathrm{~s}$. The health bar then appeared below the object for $1 \mathrm{~s}$, followed by either a motion for increases or decreases in the 
Table 1

Sample Sizes, Age-Related Descriptive Statistics, and Verbal IQ Assessed by the KBIT for Each Age Group

\begin{tabular}{|c|c|c|c|c|c|c|}
\hline & \multicolumn{2}{|c|}{ Younger children } & \multicolumn{2}{|c|}{ Older children } & \multicolumn{2}{|c|}{ Young adults } \\
\hline & Experimental & Control & Experimental & Control & Experimental & Control \\
\hline$N$ & 20 & 25 & 29 & 23 & 22 & 21 \\
\hline Sex & $9 \mathrm{~F}$ & $10 \mathrm{~F}$ & $14 \mathrm{~F}$ & $11 \mathrm{~F}$ & $16 \mathrm{~F}$ & $14 \mathrm{~F}$ \\
\hline Age/month $M(S D)$ & $59.27(6.16)$ & $62.27(8.84)$ & $85.28(9.40)$ & 91.79 (9.33) & $20.95(2.98)$ & $20.19(3.08)$ \\
\hline Age/month range & $50.63-71.33$ & $46.01-71.95$ & 71.46-101.72 & 73.59-106.94 & $18-27$ & $18-32$ \\
\hline Verbal IQ & $99.80(15.23)$ & $104.24(12.71)$ & 101.55 (18.67) & 115 (12.77) & 107.97 (6.13) & $110.61(6.31)$ \\
\hline
\end{tabular}

Note. KBIT = Kaufman's Brief Intelligence Test.

health bar for $2.5 \mathrm{~s}$ for the healthy or sick items, respectively. The increase or decrease in the health bar was always accompanied with a tone similar to that typically found in video games. A voice recording said, "Oh look, it made her healthier!" or "Oh no, it made her sick!" for the healthy and sick items, respectively. After each trial, the animation proceeded with Birdie continuing on her journey until she encountered another green diamond, which would initiate another encoding trial. The inter-trial interval (8 s) was constant across all 32 trials. The degree of change in the health bar was equated. The order of sick and healthy items was pseudo-randomized such that Birdie's health bar never depleted or filled completely.

In the control condition, everything was the same as in the experimental condition with the exception that the encoding instructions did not include the healthy/sick descriptions of the foods, or the implementation of the health bar. The encoding instructions stated, "Meet my friend Birdie. She's taking the trip to the forest. In this forest, there will be some foods she would like to eat. There will be a lot of things that Birdie will eat during her trip. So make sure you pay very close attention to the things she eats, okay? Are you ready to start?" Similar to the experimental condition, Birdie encountered the green diamonds at fixed intervals, each of which initiated an encoding trial. The presentation duration of each item was identical to that in the experimental condition. Each item was presented on the screen by itself for $5.5 \mathrm{~s}$. We implemented a neutral tone at a matched timing window to experimental condition, to ensure that the two conditions differed minimally, except for the gain/ loss framing of the studied items (see Figure 1B).

Immediately after the encoding phase of each block, children were given a self-paced three-alternative-forced-choice test phase consisting of 16 trials. The three options included a target (i.e., a studied item), and two lures (i.e., perceptually similar exemplars of the corresponding target). Children were asked to choose the exact food item that Birdie ate during her journey by pointing at one of the three options (see Figure 1C). The experimenter recorded children's responses on paper. The order of test trials was randomized. The position of the targets (left, middle, or right) was counterbalanced across test trials. All items were counterbalanced such that they were assigned as targets or lures an equal number of times across participants. Each exemplar within a triplet was assigned as the target, whereas the other two exemplars were assigned as lures, resulting in three task versions. Each of the versions was duplicated for the experimental versus control conditions, totaling six task versions. The entire experimental procedure lasted approximately 35-40 min.

Adults. To avoid potential ceiling effects in adults, the procedures differed between children and adults such that the level of difficulty increased for adults. In contrast to children who received two encoding-test blocks (i.e., 16 encoding trials followed by 16 test trials per block), the task procedure in adults consisted of a single encoding-test phase with 32 encoding and 32 test trials.

\section{Verbal Intelligence}

All children were administered the Kaufman's Brief Intelligence Test, 2nd ed. (KBIT-2; Kaufman \& Kaufman, 1990) to assess general verbal intelligence. Children were instructed to choose one image that was the best match for a word or phrase from a page showing six different images at a time (e.g., what is something that floats and you can ride in - a boat), and to respond with a one-word answer to verbal riddles (e.g., what is something that wags its tail and barks? - dog). The test, which had an increasing level of difficulty in each section, 


\section{A Encoding}

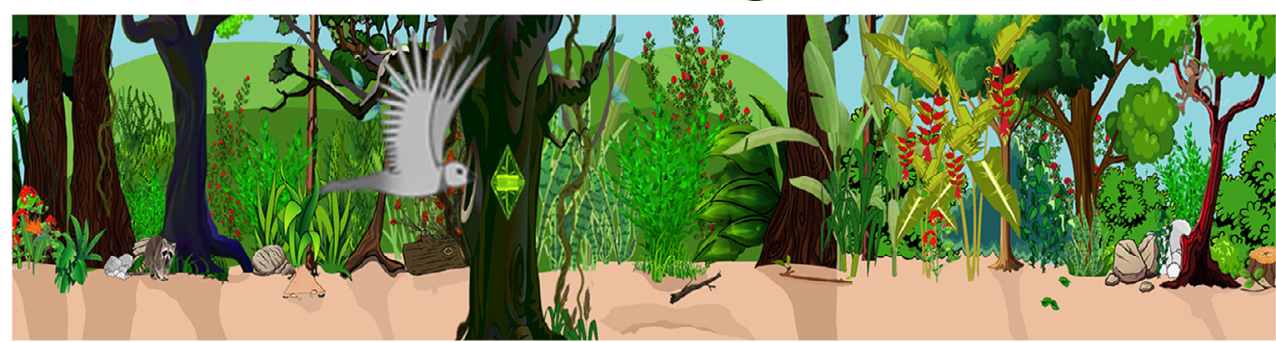

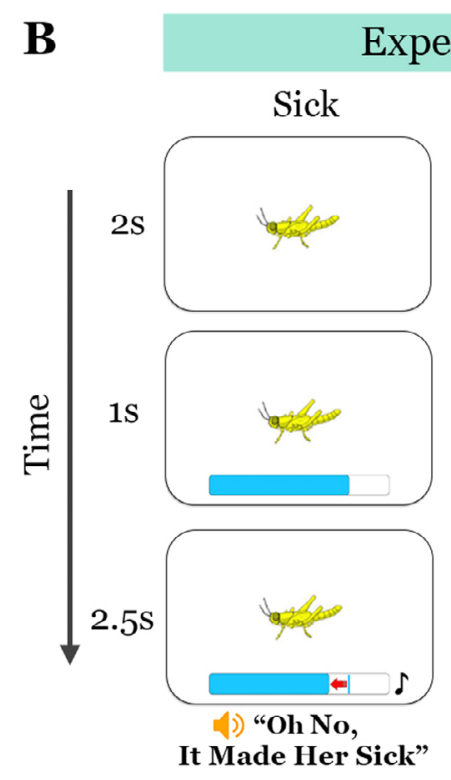

$\mathbf{C}$ mental

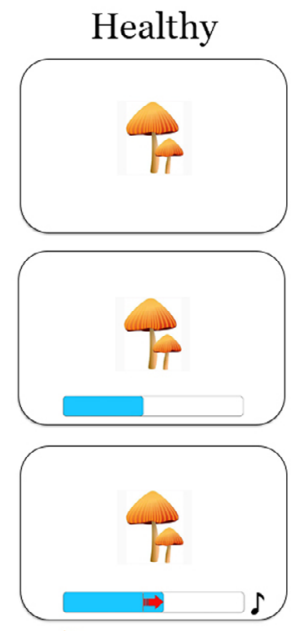

4) “Oh Look, It Made Her Healthier"

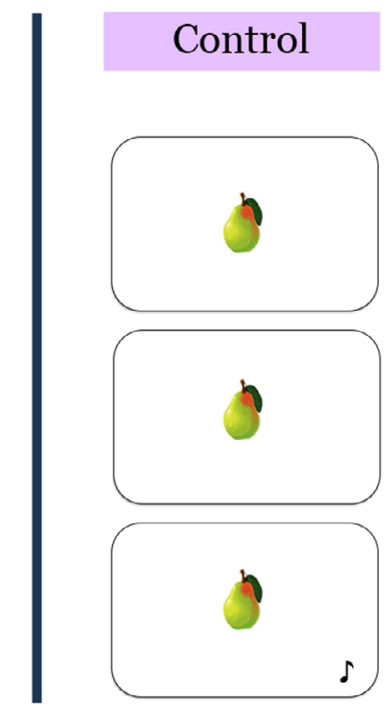

(1)

3AFC Test
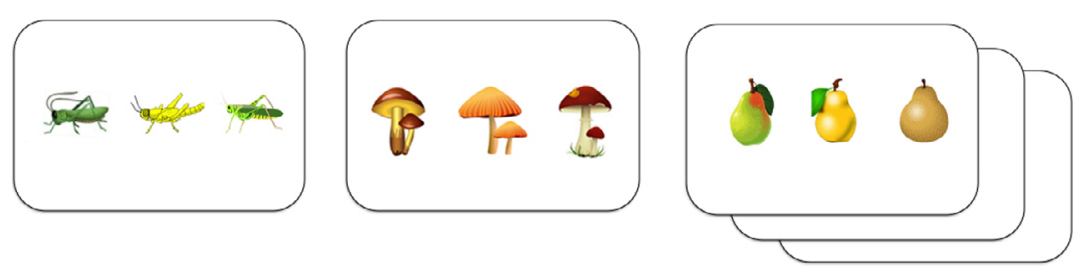

Figure 1. (A) A screenshot of the forest cartoon with Birdie and a green diamond indicating a location of the food. (B) An example of an encoding trial in the experimental and control conditions. In the experimental condition, the objects were presented for $2 \mathrm{~s}$, followed by the appearance of a "health bar" (i.e., the horizontal turquoise bar) for $1 \mathrm{~s}$. Then there was either an increase or a decrease in the health bar, accompanied by a tone for the healthy and sick trials, respectively. Note that the red arrows are only for visual illustration. In the control condition, an image was presented on its own for a matched duration. (C) Examples of the self-paced 3 alternative forced choice (AFC) test phase, which was identical between the two conditions.

was terminated when a child provided four consecutive incorrect responses. A standard score was calculated for each child based on his/her age.

Adults were administered the 45-item American National Adult Reading Test (AMNART; Grober \& Sliwinski, 1991-an American version of the
National Adult Reading Test; Nelson, 1982). This test measures participants' ability to read irregular words aloud. Pronunciation errors were tallied and AMNART-estimated IQ score was calculated using Grober and Sliwinski's formula, which accounts for years of education. 


\section{Results}

The proportion of correct trials out of 32 total test trials was calculated for each participant. We conducted several preliminary analyses. There were no sex differences in memory performance for any of the three age groups, $p>.22$. Additionally, KBIT scores did not differ between the two conditions in either group of children, all $p^{\prime} s>.63$, and the AMNART scores did not differ between the two conditions in young adults, $p=.66$. Memory performance did not differ among the three animation versions, $p=.17$, suggesting that there were no unintended differences in difficulty across task versions.

\section{The Effects of Age and Conditions on Mnemonic Discrimination}

Next, we examined the effects of age and condition on mnemonic discrimination performance using a between-subjects factorial analysis of variance (ANOVA). We found a significant main effect of age, $F(2,135)=38.81, p<.001, M S E=.59$, $\eta_{\mathrm{p}}{ }^{2}=.37$, a nonsignificant main effect of Condition, $F(1,135)=2.94, p=.09, M S E=.05, \eta_{\mathrm{p}}{ }^{2}=.02$, and a significant Age $\times$ Condition interaction, $F(2$, $135)=3.48, p=.03, M S E=.05, \eta_{p}{ }^{2}=.05$. Younger children performed better in the experimental condition compared to the control condition $(M=.62$, $S E=.04$ vs. $M=.73, S E=.03), \quad t(43)=-2.39$, $p=.02$. In contrast, older children $(M=.85$, $S E=.02$ vs. $M=.85, S E=.02), t(50)=.14, p=.89$, and adults $(M=.89, S E=.02$ vs. $M=.88$, $S E=.02), t(42)=.10, p=.92$, performed similarly in the two conditions (see Figure 2).

Importantly, although gain-loss framing enhanced mnemonic discrimination in younger children, their performance was still lower than older children in the control group, $t(41)=-3.42$, $p=.001$, suggesting that age-related differences are not abolished by gain-loss framing.

\section{The Effects of Gain Versus Loss Framing on Mnemonic Discrimination}

Next we asked whether sick and healthy items were remembered differently across three age groups within the experimental condition. A 3 (age groups) $\times 2$ (item type: sick, healthy) mixed ANOVA showed a nonsignificant Age $\times$ Item-Type interaction, $F(2,68)=0.46, p=.64, M S E=.003$, $\eta_{\mathrm{p}}{ }^{2}=.01$. There was a significant main effect of age, $F(2,68)=9.51, p<.001$ MSE $=.28, \eta_{p}{ }^{2}=.22$, such that younger children performed more poorly than their older counterparts, all $p^{\prime} \mathrm{s}<.003$, whereas older children and young adults did not differ, $p=.56$. Interestingly, there was also a main effect of Item type, $F(1,68)=11.94, \quad p=.001, \quad M S E=.09$, $\eta_{\mathrm{p}}{ }^{2}=.15$, such that mnemonic discrimination was better for sick items than healthy items (see Figure 3).

\section{Discussion}

Preschool-aged children have consistently displayed deficits in fine-grained mnemonic discrimination, showing a proclivity for mistaking similar objects for studied items (Canada et al., 2018; Ngo et al., 2018; Rollins \& Cloude, 2018). However, how nonmnemonic top-down factors such as motivational state influence children's abilities to discriminate similar memories has not been studied. This study, to our knowledge, is the first to investigate the influence of motivation through gain-loss framing on mnemonic discrimination in children. Two main findings were revealed.

\section{The Influence of Gain-Loss Framing on Mnemonic Discrimination}

First, we showed that framing items as either positive or negative boosted the youngest children in their subsequent fine-grain discrimination for learned items from similar exemplars. That is, young children who viewed objects in a gain-loss frame outperformed those who viewed objects in a traditional task variant of the MST. Importantly, this improvement did not bring the younger children's mnemonic discrimination performance on par with older children or young adults in the control condition. Thus, although there is a degree of plasticity in mnemonic discrimination that depends on the learning condition, the general age trend persists. These results demonstrate that the previously reported age effects on mnemonic discrimination using various tasks were not primarily due to factors such as the lack of motivation or age appropriateness of the task. Instead, the age-related improvements in mnemonic discrimination are likely linked to neurobiological changes in intraand extrahippocampal regions that are important for the development of pattern separation during childhood (Keresztes, Ngo, Lindenberger, WerkleBergner, \& Newcombe, 2018).

Our results on the enhancement effects of gainloss framing on mnemonic discrimination in children converge with previous findings from the 

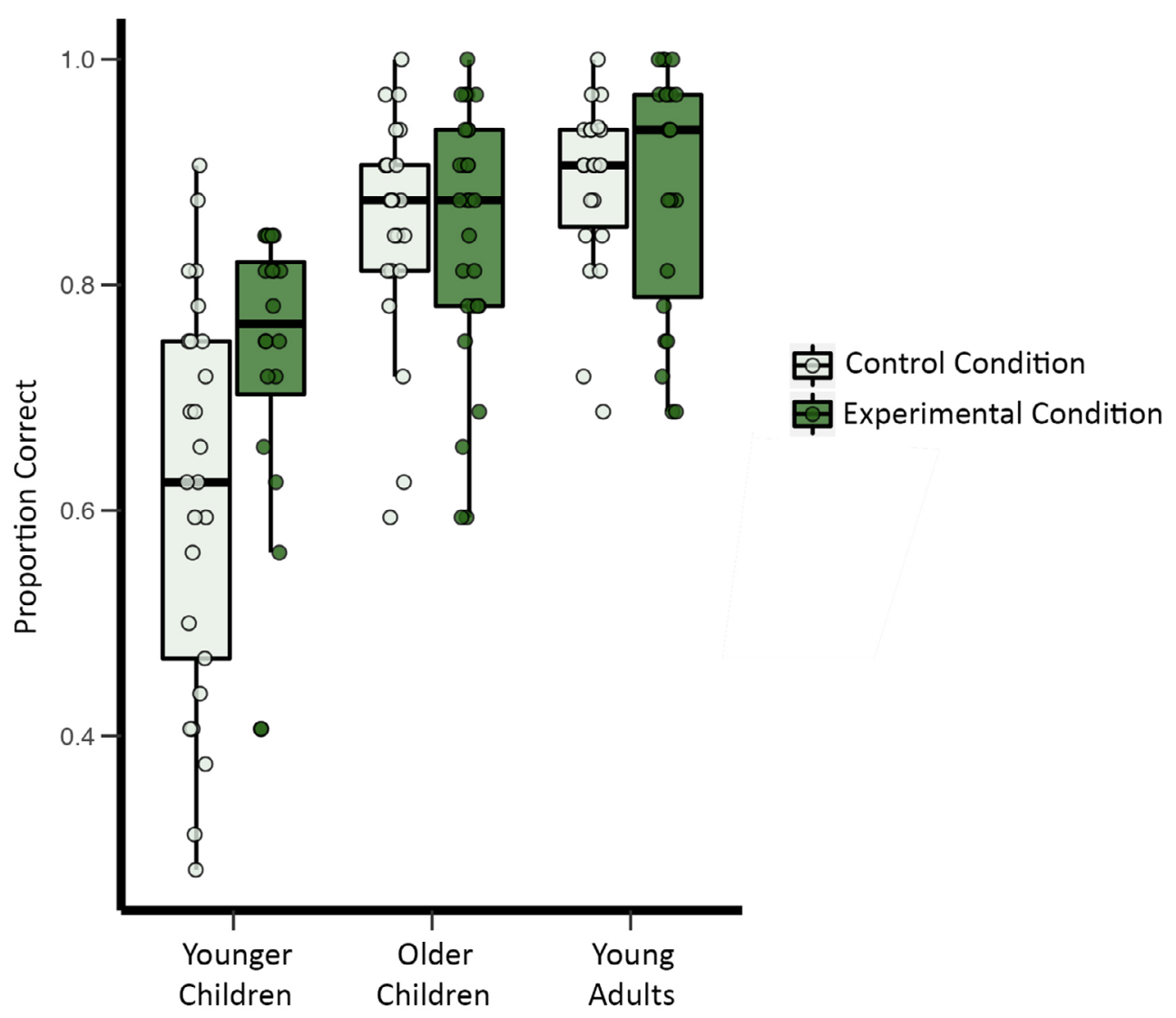

Figure 2. Accuracy distribution of the control and experimental conditions across three age groups. Black horizontal bars indicate the group medians.

adult memory literature demonstrating that mnemonic discrimination can be influenced by arousal. For example, anxiety evoked by the threat of a shock at encoding enhanced mnemonic discrimination, but the threat of a shock at retrieval impaired mnemonic discrimination in the MST (Balderston et al., 2017). In another study, arousal evoked by emotional stimuli (e.g., poisonous snake) prior to the encoding of neural stimuli enhanced subsequent mnemonic discrimination, and the degree of arousal measured by a change in salivary alpha amylase correlated with memory performance in young adults (Segal et al., 2012). The author suggested that this facilitation in pattern separation for items encoded during threat might be mediated by noradrenergic activity during encoding. In agreement with this view, a model proposed by Kassab and Alexandre (2018) predicts that neuro-modulatory signals may act in concert with cortical inputs to inform the DG about the changing demands on pattern separation under different conditions. According to this model, the hilus of the DG may serve as a convergent zone whereby bottom-up factors (i.e., pattern similarity) interact with top-down factors (i.e., motivation), which jointly determine the engagement of pattern separation.

In this study, we did not detect an enhancement effect of the gain-loss framing in older children or young adults. One possibility is that older children and young adults are able to spontaneously encode and retrieve memories with high specificity, such that they might not need the motivational aid. Another possibility is that these effects would have been observed in a more difficult task, although it is worth noting that only $3.85 \%$ and $11.36 \%$ of older children and young adults performed at ceiling level in our paradigm, respectively. The third possibility is that older children and young adults may be more susceptible to other means of motivation, such as monetary rewards (reviewed in Murty \& Dickerson, 2016), compared to the gain-loss framing employed in the current work.

\section{Asymmetric Effects of Gain Versus Loss Frame on Mnemonic Discrimination}

Second, we found asymmetric framing effects across all age groups such that negative framing 


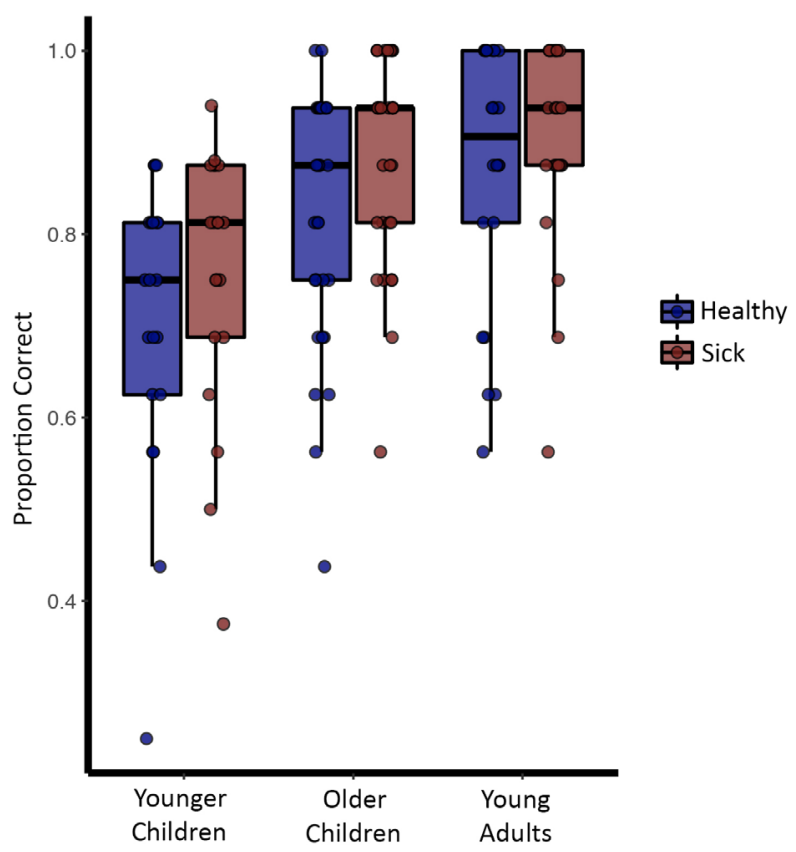

Figure 3. Accuracy distribution of the sick and healthy items in the experimental condition across three age groups. Black horizontal bars indicate the group medians.

yielded higher mnemonic discrimination accuracy than positive framing. It is likely that in addition to serving as a motivational aid, gain-loss framing also provokes emotional valence by inducing appetitive and aversive experiences at encoding.

These results align with previous findings that young adults, and older adults in some cases, show better memory for the details of negative items compared to those of neutral (Kensinger, GaroffEaton, \& Schacter, 2006, 2007a) and positive items (Kensinger, Garoff-Eaton, \& Schacter, 2007b). Similarly, the threat of receiving a painful thermal probe increased memory for individual items that were devoid of any contextual representation, whereas worsening recollection-based memory, which contains details about the relationships among features of an episode (Bauch, Rausch, \& Bunzeck, 2014). Together, this study and others suggest that memory for negatively framed items (i.e., those that might evoke an aversive valence) yields superior mnemonic discrimination compared to those that are positively framed.

\section{Limitations and Future Direction}

One limitation of the current work is that we did not measure physiological responses (e.g., galvanic skin response) or viewing behaviors, which would have provided insights into whether the gain-loss framing enhanced mnemonic discrimination through increasing attention and/or arousal at encoding. Furthermore, other approaches for assessing the role of motivational and emotional states on mnemonic discrimination in children are worth considering for future studies. First, in our design, studied items were arbitrarily assigned a positive or negative valence, as opposed to being intrinsically perceived as positive or negative. It would also be interesting to examine whether emotional stimuli (e.g., fire vs. a calming meadow, see Leal, Tighe, Jones, \& Yassa, 2014) would result in enhanced discrimination in younger children. Second, the current work did not implement motivation as a behavior-contingent-outcomes paradigm (see work by Murty, DuBrow, \& Davachi, 2015; Wittman et al., 2005). Our paradigm did not include a behavior-contingent-outcomes element because our goal was to modify the standard MST minimally, which involves an incidental encoding task (e.g., indoor/outdoor judgment). It is possible that employing a more explicit reward manipulation could result in a more robust enhancement of mnemonic discrimination. Nonetheless, although the differences between the experimental and control conditions (which are similar to those in the standard MST) were relatively subtle, they sufficiently motivated children to alter memory for the fine-grained details of the studied items.

As mentioned earlier, to test whether increased attention drives the effects of motivation on mnemonic discrimination, future research could characterize differences in viewing behavior with and without gain-loss framing. However, it is worth noting that in the current work there were more features to attend to during the encoding phase of the experimental compared to the control conditions. In the experimental condition, each item was shown simultaneously with the health bar for 3.5 of the $5.5 \mathrm{~s}$ at encoding. In contrast, in the control condition each item was presented by itself for the whole $5.5 \mathrm{~s}$ without any other features that could potentially reduce viewing behavior oriented at the to-be-remembered objects. Therefore, it is unlikely that the motivational effects on mnemonic discrimination reported here is primarily due to increased viewing behaviors. Nonetheless, future research could include eye tracking with the gain-loss framing manipulation to characterize the impact of motivation on viewing behavior at encoding.

\section{Conclusions}

In acknowledging the adaptive nature of human memory systems, it remains critical to delineate the 
influence of motivation on the core properties of episodic memory. In fact, it has been suggested that in the first few years of life, children may prioritize extracting schematic knowledge at the expense of encoding and recollecting past events with high specificity. The precedence of learning the general rules of the environment over remembering the specifics of past events may be advantageous, allowing infants and toddlers to build strong semantic knowledge of world (Keresztes et al., 2018; Newcombe, Lloyd, \& Ratliff, 2007). An important question arising from this view is whether young children could prioritize encoding items with high specificity in circumstances in which the details of past experiences would be advantageous for subsequent remembering. Evidence from this research suggests that there is a degree of malleability in mnemonic discrimination in preschoolers, such that motivation boosts mnemonic discrimination. However, motivation did not abolish the robust age-related differences between preschoolers and their older counterparts.

\section{Data Availability Statement}

Data from this experiment have been made publically available through the Open Science Framework at https://osf.io/8k43j/.

\section{References}

Atkinson, A. L., Waterman, A. H., \& Allen, R. J. (2019). Can children prioritize more valuable information in working memory? An exploration into the effects of motivation and memory load. Developmental Psychology, 55, 967-980. https://doi.org/10.1037/dev0000692

Balderston, N. L., Mathur, A., Adu-Brimpong, J., Hale, E. A., Ernst, M., \& Grillon, C. (2017). Effect of anxiety on behavioural pattern separation in humans. Cognition and Emotion, 31, 238-248. https://doi.org/10.1080/ 02699931.2015.1096235

Bauch, E. M., Rausch, V. H., \& Bunzeck, N. (2014). Pain anticipation recruits the mesolimbic system and differentially modulates subsequent recognition memory. Human Brain Mapping, 35, 4594-4606.

Berry, E. D., Waterman, A. H., Baddeley, A. D., Hitch, G. J., \& Allen, R. J. (2018). The limits of visual working memory in children: Exploring prioritization and recency effects with sequential presentation. Developmental Psychology, 54, 240-253. https://doi.org/10. $1037 /$ dev0000427

Canada, K. L., Ngo, C. T., Newcombe, N. S., Geng, F., \& Riggins, T. (2018). It's all in the details: Relations between young children's developing pattern separation abilities and hippocampal subfield volumes. Cerebral Cortex. https://doi.org/10.1093/cercor/bhy211

Ghetti, S., \& Bunge, S. A. (2012). Neural changes underlying the development of episodic memory during middle childhood. Developmental Cognitive Neuroscience, 2, 381-395. https:/ / doi.org/10.1016/j.dcn.2012.05.002

Grober, E., \& Sliwinski, M. (1991). Development and validation of a model for estimating premorbid verbal intelligence in the elderly. Journal of Clinical and Experimental Neuropsychology, 13, 933-949. https://doi.org/ $10.1080 / 01688639108405109$

Kassab, R., \& Alexandre, F. (2018). Pattern separation in the hippocampus: Distinct circuits under different conditions. Brain Structure and Function, 223, 2785-2808. https:/ / doi.org/10.1007/s00429-018-1659-4

Kaufman, A. S., \& Kaufman, N. L. (1990). Kaufman brief intelligence test. Circle Pines, MN: American Guidance Service.

Kensinger, E. A., Garoff-Eaton, R. J., \& Schacter, D. L. (2006). Memory for specific visual details can be enhanced by negative arousing content. Journal of Memory and Language, 54, 99-112. https://doi.org/10.1016/j. jml.2005.05.005

Kensinger, E. A., Garoff-Eaton, R. J., \& Schacter, D. L. (2007a). Effects of emotion on memory specificity: Memory trade-offs elicited by negative visually arousing stimuli. Journal of Memory and Language, 56, 575591. https://doi.org/10.1016/j.jml.2006.05.004

Kensinger, E. A., Garoff-Eaton, R. J., \& Schacter, D. L. (2007b). Effects of emotion on memory specificity in young and older adults. The Journals of Gerontology Series B: Psychological Sciences and Social Sciences, 62, 208215. https: / / doi.org/10.1093/geronb/62.4.P208

Keresztes, A., Bender, A. R., Bodammer, N. C., Lindenberger, U., Shing, Y. L., \& Werkle-Bergner, M. (2017). Hippocampal maturity promotes memory distinctiveness in childhood and adolescence. Proceedings of the National Academy of Sciences of the United States of America, 114, 9212-9217. https://doi.org/10.1073/pnas. 1710654114

Keresztes, A., Ngo, C. T., Lindenberger, U., WerkleBergner, M., \& Newcombe, N. S. (2018). Hippocampal maturity drives memory from generalization to specificity. Trends in Cognitive Science, 22, 676-686. https://d oi.org/10.1016/j.tics.2018.05.004

Leal, S. L., Tighe, S. K., Jones, C. K., \& Yassa, M. A. (2014). Pattern separation of emotional information in hippocampal dentate and CA3. Hippocampus, 24, 11461155. https://doi.org/10.1002/hipo.22298

Light, P. H., Buckingham, N., \& Robbins, A. H. (1979). The conservation task as an interactional setting. British Journal of Educational Psychology, 49, 304-310. https://d oi.org/10.1111/j.2044-8279.1979.tb02430.x

Murty, V. P., \& Dickerson, K. C. (2016). Motivational influences on memory. In S.-i Kim, J. Reeve, \& M. Bong (Eds.), Recent developments in neuroscience research on human motivation (pp. 203-227). Emerald Group. https:/ / doi.org/10.1108/S0749-742320160000019019 
Murty, V. P., DuBrow, S., \& Davachi, L. (2015). The simple act of choosing influences declarative memory. The Journal of Neuroscience, 35, 6255-6264. https://doi.org/ 10.1523/JNEUROSCI.4181-14.2015

Nelson, H. E. (1982). National Adult Reading Test (NART): For the assessment of premorbid intelligence in patients with dementia: Test manual. Windsor, ON: NFER-Nelson.

Newcombe, N. S., Lloyd, M. E., \& Ratliff, K. R. (2007). Development of episodic and autobiographical memory: A cognitive neuroscience perspective. Advances in Child Development and Behavior, 35, 37-85. https://doi. org/10.1016/B978-0-12-009735-7.50007-4

Ngo, C. T., Lin, Y., Newcombe, N. S., \& Olson, I. R. (2019). Building up and wearing down episodic memory: Mnemonic discrimination and relational binding. Journal of Experimental Psychology: General. https://doi. org $/ 10.1037 /$ xge0000583

Ngo, C. T., Newcombe, N. S., \& Olson, I. R. (2018). The ontogeny of relational memory and pattern separation. Developmental Science. https://doi.org/10.1111/desc.12556

Norman, K. A., \& O'Reilly, R. C. (2003). Modeling hippocampal and neocortical contributions to recognition memory: A complementary-learning-systems approach. Psychological Review, 110, 611-646. https://doi.org/10. 1037/0033-295X.110.4.611

O'Reilly, R. C., \& McClelland, J. L. (1994). Hippocampal conjunctive encoding, storage, and recall: Avoiding a trade-off. Hippocampus, 4, 661-682. https://doi.org/10. 1002/hipo.450040605
Olson, I. R., \& Newcombe, N. S. (2014). Binding together the elements of episodes: Relational memory and the developmental trajectory of the hippocampus. In P. J. Bauer \& R. Fivush (Eds.), The Wiley handbook and the development of children's memory (Vol. I/II, pp. 285-308). Chichester, UK: Wiley Blackwell.

Peterson, C., Warren, K. L., \& Short, M. M. (2011). Infantile amnesia across the years: A 2 year follow-up of children's earliest memories. Child Development, 82, 1092-1105. https://doi.org/10.1111/j.1467-8624.2011. 01597.x

Rollins, L., \& Cloude, E. B. (2018). Development of mnemonic discrimination during childhood. Learning $\mathcal{E}$ Memory, 25, 294-297. https://doi.org/10.1101/lm. 047142.117

Segal, S. K., Stark, S. M., Kattan, D., Stark, C. E., \& Yassa, M. A. (2012). Norepinephrine-mediated emotional arousal facilitates subsequent pattern separation. Neurobiology of Learning and memory, 97, 465-469. https://doi. org/10.1016/j.nlm.2012.03.010

Wittman, B. C., Schott, B. H., Guderian, S., Frey, J. U., Heinze, H. J., \& Düzel, E. (2005). Reward-related fMRI activation of dopaminergic midbrain is associated with enhanced hippocampus-dependent long-term memory formation. Neuron, 45, 459-467. https://doi.org/10. 1016/j.neuron.2005.01.010

Yassa, M. A., \& Stark, C. E. (2011). Pattern separation in the hippocampus. Trends in Neuroscience, 24, 515-525. https:/ / doi.org/10.1016/j.tins.2011.06.006 